\title{
On the Convergence of an Algorithm Computing Minimum-Norm Solutions of Ill-Posed Problems
}

\author{
By J. T. Marti
}

\begin{abstract}
The paper studies a finite element algorithm giving approximations to the minimum-norm solution of ill-posed problems of the form $A f=g$, where $A$ is a bounded linear operator from one Hilbert space to another. It is shown that the algorithm is norm convergent in the general case and an error bound is derived for the case where $g$ is in the range of $A A^{*}$. As an example, the method has been applied to the problem of evaluating the second derivative $f$ of a function $g$ numerically.
\end{abstract}

1. Introduction. Let $X$ and $Y$ be real Hilbert spaces and $A$ a bounded linear operator from $X$ into $Y$. The problem of determining, for given $g$ in the range $A(X)$ of $A$, solutions $f$ in $X$ of

$$
A f=g
$$

is said to be ill-posed if $A$ has no continuous inverse on $A(X)$. If there are more than one solutions of (1) one is frequently interested in the solution which has minimum norm in $X$, the minimum-norm solution $f_{0}$ of (1). The existence and uniqueness of $f_{0}$ is a consequence of the well-known result (see e.g. [6, p. 124]) on the existence of a unique nearest point to the origin of $X$ in every closed convex set in $X$. Solving (1) for $g$ not in the closure of the range of $A$, in the sense that the solution $f$ has to satisfy $\|A f-g\|=\min \{\|A h-g\|: h \in X\}$, is easily seen to be equivalent to the problem of solving $A f=P g$, where $P$ is the orthogonal projection from $Y$ onto the closure $\overline{A(X)}$ of $A(X)$ in $Y$. There are numerous examples of problems of the form (1), e.g. Fredholm integral equations of the first kind, where the mathematical formulation of a host of applications is leading to such equations (see e.g. [13] or [14]).

Usually, ill-posed problems are extremely difficult to solve numerically. Several schemes leading to approximate solutions of (1) have been proposed, e.g. the collocation method (e.g. in [1], [2], [4]) or Tikhonov's method [3], [10], [11], [12] . But up to now, little is known on the convergence of both methods in the general case (e.g. if $A$ does not possess an inverse), as on the crucial choice of regularization parameters occurring in Tikhonov's method. The reason for the lack of a convergence proof for the first method lies in the fact that there are still no results available on the influence of the collocation errors in solving (1) by this method.

Received November 16, 1978.

AMS (MOS) subject classifications (1970). Primary 65R05; Secondary 65F20.

Key words and phrases. Ill-posed problem, algorithm, minimum-norm solution, Fredholm integral equation of the first kind. 
In this paper we consider an algorithm for solving (1) numerically. This algorithm, giving the minimum-norm solution of (1), has been proposed in [7], [8]. The algorithm has no restrictions on the operator $A$ (such as the existence of an inverse of $A$, a condition which is often required for the Tikhonov method; see e.g. [12, p. 45] or [3, p. 870]). Furthermore, the algorithm studied in this article uses a technique whose computational part has some similarities with Galerkin's method, though it is conceptionally much different from Galerkin's and also from Tikhonov's methods. An upper bound for the rate of convergence is derived for the restricted class of problems (1), where $A$ is an arbitrary bounded linear operator and $g$ is in the range of $A A^{*}, A^{*}$ : $Y \rightarrow X$ being the adjoint operator of $A$. More generally, if $g$ is in the range of $A$, then a convergence proof for the algorithm follows as a corollary. In case of Hermitian operators $A\left(X=Y, A^{*}=A\right)$ an upper bound for the error norm in computing the minimum-norm solution of (1) has been derived in [7] for $g$ in the range of $A^{2}$ and a convergence proof has been given for the case of arbitrary $A$ and $g$ in [8].

2. Description of the Algorithm. Let $\left\{P_{n}\right\}$ be a sequence of orthogonal projections of finite rank in $X$ such that

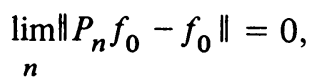

where $f_{0}$ is the minimum-norm solution of (1). The above condition is obviously satisfied if the sequence $\left\{P_{n}(X)\right\}$ is increasing and the union of the sets $P_{n}(X)$ is dense in $X$, e.g. if the $P_{n}$ are the projections associated with an orthonormal basis $\left\{u_{1}, \ldots\right.$, $\left.u_{n}\right\}$ of $X$, where $P_{n} v=\Sigma_{i=1}^{n}\left(v, u_{i}\right) u_{i}, v \in X$. Several well-known bounds for $\| P_{n} f_{0}-$ $f_{0} \|$ are available in the literature, e.g.

$$
\left\|P_{n} f_{0}-f_{0}\right\|=O\left(h^{k}\right)
$$

if $f_{0}$ has an absolutely continuous $(k-1)$ th derivative, the $k$ th derivative of $f_{0}$ is in $L_{2}[a, b]$ and $P_{n}\left(L_{2}[a, b]\right)$ is the set of polynomial spline functions of odd degree $\geqslant k-1$ with knots $a, a+h, a+2 h, \ldots, b-h, b$ (see e.g. [9], where a proof is given for the case $k=2$ and 4 ).

If the sequence $\left\{a_{n}\right\}$ is given by

$$
a_{n}=\inf \left\{\|A f-g\|: f \in P_{n}(X)\right\}
$$

and the sequence of positive numbers $\left\{b_{n}\right\}$ is chosen such that

$$
\left\|P_{n} f_{0}-f_{0}\right\|=o\left(b_{n}\right) \text { and } \quad b_{n}=o(1)
$$

then an algorithm for solving (1) is established by the construction of a sequence $\left\{f_{n}\right\}$ such that

$$
f_{n} \in S_{n} \quad \text { and } \quad\left\|f_{n}\right\|=\inf \left\{\|f\|: f \in S_{n}\right\} \text {, }
$$

where $S_{n}$ is the intersection of the sets $P_{n}(X)$ and $f_{0}+\left(a_{n}+b_{n}\right) A^{-1}(U)$ and $U$ is the unit ball of $X$. The existence of a unique element $f_{n}$ again is a consequence of the fact that each $S_{n}$ is a closed convex set in $X$, having a unique nearest point to the 
origin of $X$. If $\left\{v_{1}, \ldots, v_{m(n)}\right\}$ is any basis for $P_{n}(X)$ it is clear that the vector $x_{n}$ of basis coefficients $x_{n 1}, \ldots, x_{n m(n)}$ of $f_{n}$ can be computed by evaluating

$$
a_{n}=\inf \left\{\left[y^{T} B_{n} y-2 w_{n}^{T} y+(g, g)\right]^{1 / 2}, y \in \mathbf{R}^{m(n)}\right\}
$$

and by solving

$$
x_{n}^{T} M_{n} x_{n}=\inf \left\{x_{n}^{T} M_{n} x_{n}: x_{n} \in \mathbf{R}^{m(n)}, x_{n}^{T} B_{n} x_{n}-2 w_{n}^{T} x_{n}+(g, g)=\left(a_{n}+b_{n}\right)^{2}\right\}
$$

for $x_{n}$, where $M_{n}$ is the Gramian $\left[\left(v_{i}, v_{j}\right)\right]$ of the above basis and $B_{n}$ and $w_{n}$ are given by

$$
B_{n}=\left[\left(A v_{i}, A v_{j}\right)\right], \quad w_{n}=\left(\left(A v_{1}, g\right), \ldots,\left(A v_{m(n)}, g\right)\right)^{T} .
$$

We recognize $B_{n}$ as the so-called stiffness matrix of the finite element method. It is easy to verify that the matrix $B_{n}+\lambda M_{n}$ is (Hermitian and) positive definite for all positive numbers $\lambda$. Now, it follows that $x_{n}$ is the solution $x$ of the system of linear equations

$$
\left(B_{n}+\lambda M_{n}\right) x=w_{n},
$$

obtainable by the Cholesky method, where $\lambda$ is the unique positive solution [7] of the scalar equation

$$
-\lambda x^{T} M_{n} x-w_{n}^{T} x+(g, g)-\left(a_{n}+b_{n}\right)^{2}=0 .
$$

Here, Newton's method is applicable, since the derivative of the left-hand side of the above equation, as a function of $\lambda$, is $2 \lambda x^{T} M_{n}\left(B_{n}+\lambda M_{n}\right)^{-1} M_{n} x$ and this expression, being the $2 \lambda$ multiple of the square of the Euclidean norm of an $m(n)$-vector, is nonnegative.

Summing up, the sequence $\left\{f_{n}\right\}$ may be computed numerically by solving (3) for $x$ and $\lambda$ and then taking $f_{n}=\sum_{i=1}^{m(n)} x_{n i} v_{i}$, where $x_{n}=x$.

3. An Upper Bound for the Convergence Rate. For a large class of problems the following theorem shows that the ultimate convergence rate for solving (1) by the algorithm (2) is almost of the order of the square root of the error norm of the best approximation in $P_{n}(X)$ to the minimum-norm solution $f_{0}$ of (1).

THEOREM. For every $g$ in $A A^{*}(Y)$ the error norm for the algorithm (2) has the property that

$$
\left\|f_{n}-f_{0}\right\|=O\left(b_{n}^{1 / 2}\right)
$$

where $f_{0}$ is the minimum-norm solution of (1).

Proof. Since by (2b) $\left\|P_{n} f_{0}-f_{0}\right\|=o\left(b_{n}\right)$ there is, in view of (1), an integer $m$ such that

$$
\left\|A P_{n} f_{0}-g\right\| \leqslant\|A\|\left\|P_{n} f_{0}-f_{0}\right\| \leqslant a_{n}+b_{n}, \quad n \geqslant m .
$$

Thus

$$
P_{n} f_{0} \in f_{0}+\left(a_{n}+b_{n}\right) A^{-1}(U), \quad n \geqslant m,
$$


which by (2c) implies that

$$
\left\|f_{n}\right\| \leqslant\left\|P_{n} f_{0}\right\| \leqslant\left\|f_{0}\right\|, \quad n \geqslant m .
$$

Since $A f_{0}=g=A A^{*} h$ for some $h$ in $Y$ and since by a known theorem [5] $f_{0}$ is in the closure $\overline{A^{*}(Y)}$ in $X$ of the range of $A^{*}$, one has

$$
f_{0}-A^{*} h \in \overline{A^{*}(Y)} \cap A^{-1}(0)
$$

It is well known that the above intersecting sets are orthogonal. Therefore,

$$
f_{0}=A^{*} h
$$

By a twofold application of the Pythagorean theorem it is now easy to see that

$$
\left\|f_{n}-f_{0}\right\|^{2} \leqslant 2\left(f_{0}-f_{n}, f_{0}\right)
$$

By $(2 c)$ and (2a) this yields

$$
\begin{aligned}
1 / 2\left\|f_{n}-f_{0}\right\|^{2} & \leqslant \sup \left\{\left(f, f_{0}\right): A f \in\left(a_{n}+b_{n}\right) U\right\} \\
& =\left(a_{n}+b_{n}\right) \sup \{(A f, h): A f \in U\} \\
& \leqslant\|h\|\left[b_{n}+\inf \left\{\left\|A\left(f-f_{0}\right)\right\|: f \in P_{n}(X)\right\}\right] \\
& \leqslant\|h\|\left(b_{n}+\|A\|\left\|P_{n} f_{0}-f_{0}\right\|\right), \quad n \geqslant m .
\end{aligned}
$$

Due to $(2 b)$ one then has the estimate

$$
\left\|f_{n}-f_{0}\right\|^{2} \leqslant 2\|h\| b_{n}(1+o(1)), \quad n \geqslant m,
$$

hence

$$
\left\|f_{n}-f_{0}\right\|=O\left(b_{n}^{1 / 2}\right)
$$

Corollary. For every $g$ in $A(X)$ the sequence $\left\{f_{n}\right\}$ generated by the algorithm (2) converges in $X$ to the minimum-norm solution $f_{0}$ of (1).

Proof. Let $\left\{f_{k(n)}\right\}$ be any subsequence of $\left\{f_{n}\right\}$ given by (2). Since by (4), $f_{n} \in$ $U, n \geqslant m$ and since $U$ is weakly sequentially compact in $X$, there is a subsequence of $\left\{f_{k(n)}\right\}$, which we also denote by $\left\{f_{k(n)}\right\}$, converging weakly to an element, say $f$ of $U$. Clearly (5) shows that

$$
\underset{n}{\lim \sup }\left\|f_{k(n)}-f_{0}\right\| \leqslant 2\left(f_{0}-f, f_{0}\right)
$$

Next, using (2c), (2a) and (2b), one obtains

$$
\begin{aligned}
\left(f_{0}-f, A^{*} h\right) & =\lim _{n}\left(f_{0}-f_{k(n)}, A^{*} h\right) \\
& =\lim _{n}\left(g-A f_{k(n)}, h\right) \leqslant \underset{n}{\| \lim _{n}\left(a_{n}+b_{n}\right)} \\
& \leqslant\left\|_{n} h\right\| \lim _{n}\left(\|A\|\left\|P_{n} f_{0}-f_{0}\right\|+b_{n}\right)=0, \quad h \in Y .
\end{aligned}
$$


Since $f_{0}$ is in the closure of $A^{*}(Y)$ in $X$ it then follows that

$$
\left(f_{0}-f, f_{0}\right)=0
$$

and finally by (6) that

$$
\underset{n}{\lim \| f_{k(n)}}-f_{0} \|=0
$$

Finally, since we have shown that any subsequence of $\left\{f_{n}\right\}$ has a subsequence which is norm convergent to $f_{0}$, the proof of the corollary is complete.

4. The Example of Computing the Second Derivative. The second derivative $f$ of a function $g$ satisfies the well-known Fredholm integral equation of the first kind

$$
\int_{0}^{s}(s-t) f(t) d t=g(s)-g(0), \quad s \geqslant 0 .
$$

Defining the integral operator $K$ from $L_{2}[0,1]$ into itself with kernel

$$
k(s, t)= \begin{cases}s-t, & t \leqslant s, \\ 0, & t>s,\end{cases}
$$

the above integral equation obviously has the form (1), $K f=g$, for all $g$ with $g(0)=$ 0 . It is clear that $K$ is compact. The following argument shows that $K$ is injective on $L_{2}[0,1]$ :

Let $f$ be in $K^{-1}(0)$. Then, since $f$ is integrable on $[0,1],(K f)(s)=0, s \in[0,1]$, and for $s$ in $(0,1)$ and sufficiently small $h$,

$$
\begin{aligned}
\int_{0}^{s} f(t) d t & =h^{-1}\left[(K f)(s+h)-\int_{s}^{s+h}(s+h-t) f(t) d t-(K f)(s)\right] \\
& =-\int_{s}^{s+h} h^{-1}(s+h-t) f(t) d t .
\end{aligned}
$$

This implies

$$
\left|\int_{0}^{s} f(t) d t\right| \leqslant \lim _{h \rightarrow 0} \int_{s}^{s+h}|f(t)| d t=0, \quad s \in(0,1)
$$

and thus $f=0$ a.e. on $[0,1]$.

Now, since $K$ is injective, there is only one solution of $K f=g$ for every $g$ in the range of $K$. Therefore, choosing

$$
g(s)=\left(s^{4}-4 s^{3}+6 s^{2}\right) / 24, \quad 0 \leqslant s \leqslant 1,
$$

the function $f$ given by

$$
f(t)=\left(t^{2}-2 t+1\right) / 2, \quad 0 \leqslant t \leqslant 1,
$$

is the minimum-norm solution $f_{0}$ of $K f=g$.

For the numerical computations we assume $P_{n}\left(L_{2}[0,1]\right)$ to be the set of spline functions of degree one with knots $0,1 / n, 2 / n, \ldots, 1$ with a basis $\left\{v_{1}\right.$, $\left.\ldots, v_{n+1}\right\}$ of $B$-splines satisfying $v_{i+1}(j / n)=\delta_{i j}$ (the Kronecker symbol), $0 \leqslant i$, $j \leqslant n$. The advantage of such a basis (of finite elements) is the minimality of the 
support of each $v_{i}$ (being of length $\leqslant 2 / n$ ) which facilitates the numerical integration of the elements of $B_{n}$ and $w_{n}$. Here, the mass matrix $M_{n}$ is tridiagonal and can be evaluated exactly, the $i j$ th elements of $6 n M_{n}$ are 1 for $i \neq j, 2$ for $i=j=$ 1 or $n+1$ and 4 for $2 \leqslant i=j \leqslant n$. For the evaluation of $K v_{i}$ at desired points, use has been made of two-point Gaussian quadrature on each subinterval of length $1 / n$. On the other hand, the scalar products occurring in the definition of $B_{n}$ and $w_{n}$ have been computed by the trapezoidal rule with nodes coinciding with the above $n+1$ knots. The basis $\left\{v_{1}, \ldots, v_{n+1}\right\}$ has the property that $\| P_{n} f_{0}-$ $f_{0} \|=O\left(n^{-2}\right)$. In general, if $g$ is in the range of $K K^{*}$ (the hypothesis of the convergence theorem in Section 3) or in the range of $K^{2}$, then we still have the same property. Therefore, it is justified to take, e.g. $b_{n}=10^{-3} n^{-2} \log n$, where $n$ runs over the higher powers of 2 . The expected convergence rate based on this choice of $b_{n}$ is, according to the theorem in Section 3, $O\left(n^{-1} \log n\right)$. Finally, the following Table 1 gives the error norms $\left\|f_{n}-f_{0}\right\|$ for a number of approximations $f_{n}$ for $f_{0}$, computed by algorithm (2), i.e. by its numerical variant described in Section 2. Table 1 also indicates the ratio of $\left\|f_{n}-f_{0}\right\|$ to $n^{-1} \log n$.

TABLE 1

\begin{tabular}{ccc}
\hline$n$ & $\left\|f_{n}-f_{0}\right\|$ & $\left\|f_{n}-f_{0}\right\| /\left(n^{-1} \log n\right)$ \\
\hline 4 & $2.1 E-3$ & $6.0 E-3$ \\
8 & $1.0 E-3$ & $3.8 E-3$ \\
16 & $4.9 E-4$ & $2.8 E-3$ \\
32 & $2.3 E-4$ & $2.1 E-3$ \\
64 & $1.1 E-4$ & $1.7 E-3$ \\
\hline
\end{tabular}

Seminar für Angewandte Mathematik

Eidgenössische Technische Hochschule Zürich, Switzerland

1. P. M. ANSELONE, Collectively Compact Operator Approximation Theory, PrenticeHall, Englewood Cliffs, N. J., 1971.

2. K. E. AT KINSON, A Survey of Numerical Methods for the Solution of Fredholm Integral Equations of the Second Kind, SIAM, Philadelphia, Pa., 1976.

3. J. N. FRANKLIN, “On Tikhonov's method for ill-posed problems," Math. Comp., v. 28, 1974 , pp. 889-907.

4. J. GRAVES \& P. M. PRENTER, "Numerical iterative filters applied to first kind Fredholm integral equations," Numer. Math., v. 30, 1978, pp. 281-299.

5. W. J. KAMMERER \& M. Z. NASHED, "Iterative methods for best approximate solutions of linear integral equations of the first and second kinds," J. Math. Anal. Appl., v. 40, 1972, pp. $547-573$.

6. J. T. MARTI, Konvexe Analysis, Birkhäuser Verlag, Basel, 1977.

7. J. T. MARTI, On the Numerical Computation of Minimum Norm Solutions of Fredholm Integral Equations of the First Kind Having a Symmetric Kernel, Report 78-01, Seminar für Angew. Math., ETH, Zurich, 1978. 
8. J. T. MARTI, “An algorithm for computing minimum norm solutions of Fredholm integral equations of the first kind," SIAM J. Numer. Anal., v. 15, 1978, pp. 1071-1076.

9. M. H. SCHULTZ, Spline Analysis, Prentice-Hall, Englewood Cliffs, N. J., 1973.

10. A. N. TIKHONOV, "Solution of incorrectly formulated problems and the regularization method," Soviet Math. Dokl., v. 4, 1963, pp. 1035-1038.

11. A. N. TIKHONOV, "Regularization of incorrectly posed problems," Soviet Math. Dokl., v. 4, 1963, pp. 1624-1627.

12. A. N. TIKHONOV \& V. Y. ARSENIN, Solutions of Ill-Posed Problems, Winston, Washington, D. C., 1977.

13. S. TWOMEY, "The application of numerical filtering to the solution of integral equations encountered in indirect sensing measurements," J. Franklin Inst., v. 279, 1965, pp. 95-105.

14. V. VEMURI \& FANG-PAI CHEN, "An initial value method for solving Fredholm integral equations of the first kind," J. Franklin Inst., v. 297, 1974, pp. 187-200. 